\title{
Recurrent myocardial infarction caused by peripartum left main spontaneous coronary artery dissection, extending to the left anterior descending artery and left circumflex artery
}

\author{
Géraldine Godin, Juan F. Iglesias, Stéphane Noble, Marco Roffi, Sophie Degrauwe
}

Department of Cardiology, Geneva University Hospital, Geneva, Switzerland

Spontaneous coronary artery dissection (SCAD) is an important cause of myocardial infarction (MI) in otherwise healthy young and middle-aged patients without cardiovascular risk factors. Herein, is reported a multiparous (5 pregnancies, 5 deliveries) 45-year-old woman without cardiovascular risk factors who presented to the emergency room, 3 months after her last delivery, for oppressive retrosternal chest pain irradiating in the left arm. Electrocardiogram demonstrated negative $T$ waves from $\mathrm{V}_{2}-\mathrm{V}_{6}$, I, II and avL. High sensitivity troponins were elevated at $293 \mathrm{ng} / \mathrm{L} \rightarrow 1345 \mathrm{ng} / \mathrm{L}$. Coronary angiogram demonstrated left main SCAD (type 2) with extension to the distal left anterior descending artery (LAD) and the proximal circumflex artery (LCx). The right coronary artery demonstrated no signs of SCAD (Fig. 1A-C). The patient did not present a trigger factor for SCAD. Echocardiography showed moderate left ventricular dysfunction (ejection fraction 45-50\%), with apical akinesia and infero-septal hypokinesia. In the presence of conserved antegrade flow, conservative treatment was performed, and the patient was started on dual antiplatelet therapy by means of acetylsalicylic acid $100 \mathrm{mg} / \mathrm{d}$ and clopidogrel
$75 \mathrm{mg} / \mathrm{d}$. Heart failure therapy with angiotensin-converting enzyme inhibitors and beta-blockers was initiated. Forty-eight hours after the initial angiogram the patient presented recurrent retrosternal chest pain. Electrocardiogram showed negative $T$ waves from $V_{2}-V_{6}$, as well as in leads II, III and avF and troponins were elevated at $477 \mathrm{~g}$ $\rightarrow 3775 \mathrm{ng} / \mathrm{L}$, confirming recurrent MI. Repeat coronary angiogram demonstrated the previously seen spontaneous coronary artery dissections (type 2) with progression of the dissection of the circumflex artery to the distal segment of the vessel, associated with occlusion of the distal LCx (Fig. 1D-F). In the absence of hemodynamic instability, it was decided to treat the LCx dissection conservatively. Clinical evolution was favourable at 6 month follow-up. On echocardiography persistence of apical akinesia was visualized, with a slight left ventricular dysfunction (ejection fraction 50\%). Control angiogram demonstrated complete healing of the left main, left circumflex artery and left anterior descending artery (Fig. 1G-I). Peripartum associated SCAD represent a high-risk subset of patients, is associated with high-risk clinical presentation and increased cardiovascular morbidity.

Conflict of interest: None declared

Address for correspondence: Sophie Degrauwe, MD, Department of Cardiology, Rue Gabrielle-Perret-Gentil 4, 1205 Genève, Switzerland, tel: +41 7955302 94, fax: +4122 37272 29, e-mail: sophie.degrauwe@hcuge.ch 


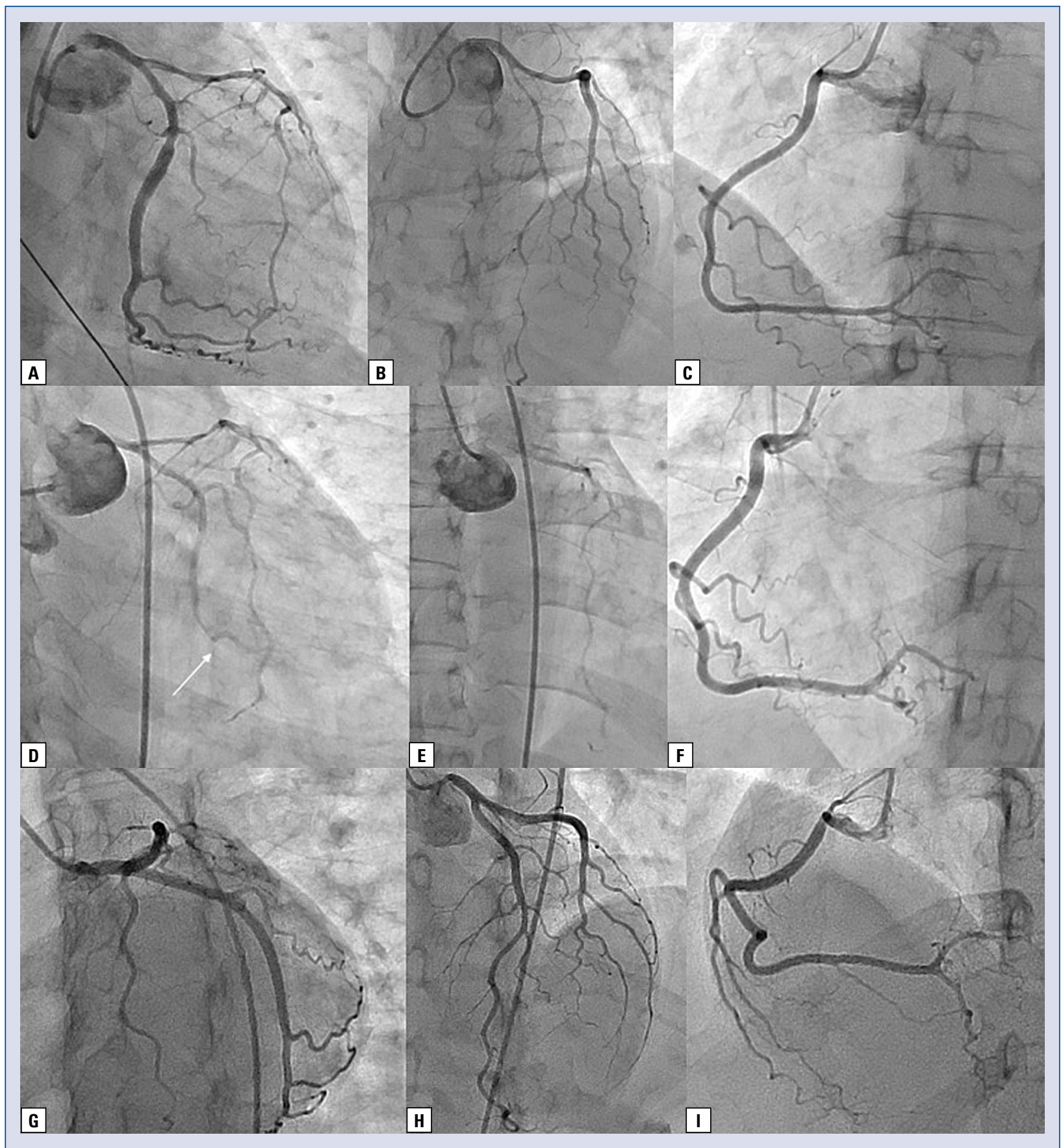

Figure 1. A-C. Initial presentation: A. Spontaneous coronary artery dissection (SCAD) of the proximal segment of the left circumflex artery; B. Spontaneous coronary artery dissection of the left anterior descending artery; C. Right coronary artery; D-F. Recurrent SCAD: D. Dissection of the proximal to distal segment of the left circumflex artery, with occlusion of the distal vessel (arrow); E. Spontaneous coronary artery dissection of the left anterior descending artery; F. Right coronary artery; G-I. Six month control coronary angiogram: G. Left circumflex artery demonstrating complete healing; H. Left anterior descending artery demonstrating complete healing; I. Right coronary artery. 